\title{
Cognitively automated assembly processes: a simulation based evaluation of performance
}

\author{
Marcel Ph. Mayer*, Barbara Odenthal, Marco Faber and Christopher M. Schlick \\ Institute for Industrial Engineering and Ergonomics at RWTH Aachen University, \\ Bergdriesch 27, 52064 Aachen, Germany
}

\begin{abstract}
The numerical control of an experimental assembly cell with two robots - termed a cognitive control unit (CCU) - is able to simulate human information processing at a rule-based level of cognitive control. To enable the CCU to work on a large range of assembly tasks expected of a human operator, the cognitive architecture SOAR is used. The CCU can plan assembly processes autonomously and react to ad-hoc changes in assembly sequences effectively. Extensive simulation studies have shown that cognitive automation based on SOAR is especially suitable for random parts supply, which reduces planning effort in logistics. Conversely, a disproportional increase in processing time was observed for deterministic parts supply, especially for assemblies containing large numbers of identical parts. In this contribution, the effect of phase-shifts in deterministic part supply is investigated for assemblies containing maximal different parts. It can be shown that the concept of cognitive automation is as well suitable for these planning problems.
\end{abstract}

Keywords: Cognitive automation, SOAR, Assembly, Joint cognitive systems

\section{Introduction}

Today one must conclude that especially in highwage countries the level of automation of many production systems has already been taken far without paying sufficient attention to the specific knowledge, skills and abilities of the human operator. According to the law of diminishing returns that kind of naive increase in automation will likely not lead to a significant increase in productivity but can also have adverse effects. Following Kinkel et al. [1] the amount of process errors is on average significantly reduced by automation, but the severity of potential consequences of a single error increases disproportionately. These "ironies of automation" [2] can be considered a vicious circle [3], where a function that was allocated to a human operator due to poor human reliability is automated. This automation results in higher function complexity, ultimately increasing the cognitive loads of the human operator for planning, teaching and monitoring, and hence leading to a more error-prone system. To reduce the error potential one could again extend automation and reinforce the vi- cious circle. During the first iteration it is quite likely that the overall performance of an automated system will increase, but the potential risk taken is often severely underestimated. Additional iterations usually deteriorate performance and lead to poor system robustness. The novel concept of cognitive automation by means of simulation of human cognition aims at breaking this vicious circle. Based on simulated cognitive functions, technical systems shall not only be able to (semi-)autonomously carry out manufacturing planning, adapt to changing supply conditions and be able to learn from experience but also to simulate goal-directed human behavior and therefore significantly increase the conformity with operator expectations. Furthermore, cognitive automation of production systems offers a technology that can efficiently and robustly automate product families with large numbers of variants enabling customer-oriented mass production.

\footnotetext{
*Corresponding author. E-mail: m.mayer@iaw.rwth-aachen.de
} 


\section{Cognitive control unit}

In order to validate the aforementioned theory, an experimental assembly cell was designed [4] and a manufacturing scenario was developed. The scenario is as follows: An engineer has designed a mechanical part of medium complexity by composing it e.g. with a CAD-system containing any number of subparts. The task for the assembly cell's cognitive control unit (CCU) is to autonomously develop and execute an efficient assembly sequence on the basis of the CAD model using the given technical resources in terms of robots, manipulators, changing devices, supplied subparts etc.

With regard to designing and developing automated robotic systems, numerous architectures have been proposed as basic structures for simulating cognitive functions [5],[6]. These software architectures combine a deliberative part for the actual planning process (planning level) with a reactive part for direct control (action level). A widely used approach here is the three-layer model that comprises cognitive, associative and reactive control layers [7], [8]. The lowest layer (reactive) contains the components that control information processing, and is designed to influence system behavior in such a way as to ensure that the required reference variables are achieved quickly and accurately. The associative layer monitors and controls the system. The majority of rule-based auxiliary functions for automation - like process control, monitoring processes and emergency processes, and adaptation routines for improving system behavior - are all embedded here. In this top layer, the system can apply "reflexive" methods (e.g. planning and learning processes, model-oriented optimization processes and knowledge-based systems) to use knowledge about itself and its environment to improve its own behavior.

Russel \& Norwig's three-layer model [7], was chosen as the basic framework for the architecture of the assembly cell's CCU. However, in order to satisfy the demands of a holistic consideration of the human-machine system, the classic three-layer architecture was expanded to include further layers and modules.

The planning layer (deliberative layer) operates on a high abstraction level with symbolic problem definitions and must satisfy only soft real-time demands. The reactive layer, on the other hand, has to monitor machine-related control loops in "hard real-time". The coordination layer (executive layer) mediates between these two layers. This is where abstract in- structions from the planning layer are transformed into concrete machine control commands. In the reverse direction, the information from the various sensors is aggregated to form an overall picture of the situation and is transmitted to the planning layer as a basis for decisions. The presentation layer as the most important extension to the classic three-layer approach forms the interface for interactive goal definition and description of the task, as well as the presentation of the current internal state of the CCU to the human operator. The knowledge module contains the knowledge base of the CCU in the form of production rules. A more detailed description can be found in [9].

The CCU as presented by Mayer et al. [10] uses the cognitive architecture SOAR [11] to simulate cognitive functions. As outlined by Mayer et al. [12], it is crucial for the human operator to understand the plan of the CCU to supervise the robotic assembly cell. Therefore, the question arises how the symbolic representation of the knowledge base of the $\mathrm{CCU}$ must be designed to ensure the conformity with the operator's expectations. Proprietary programming languages that are used in conventional automation have to be learned domain specific and do not necessarily match the mental model of the human operator. In terms of a human centered description for matching the process knowledge to the mental model, one promising approach in this particular manufacturing scenario is the use of motion descriptors, since motions are familiar to the human operator from manually performed assembly tasks [13]. These motions are also easy to anticipate in human-robot interaction. Hence, already established methods or taxonomies for manual process planning should be used. In manual assembly, it is best practice to break down complex handling tasks into fundamental motion elements. To do so the very popular MTM-1 system as a library of fundamental movements was chosen to define the motion descriptors that can be used by the $\mathrm{CCU}$ to plan and execute the robotic assembly process [14]. Further information regarding the CCU can be found e.g. in [9],[15].

\section{Simulation study}

One of today's challenges in manufacturing is the increasing complexity of assembly processes due to an increasing number of products that have to be assembled in a large variety in production space [16]. There are numerous formal approaches to solving 
planning tasks in different fields of application. Hoffmann [17] developed the Fast-Forward Planner, which is capable of deriving actions for given problems in deterministic operational areas. By contrast, other planners can handle uncertainty [18],[19]. All the planners mentioned are based on a symbolic knowledge representation. In the case of assembly planning, which requires the geometric relationship between conditions and their transitions to be adequately represented, this kind of knowledge representation becomes extremely complex, even for simple tasks.

Whereas in conventional automation each additional product or variant significantly increases the organizational overhead, cognitively automated assembly cells are theoretically able to autonomously plan, execute and replan the expected tasks on the basis of a digital model of the product to be assembled in conjunction with a set of production rules. No explicit knowledge on how to solve the assembly problem is needed. To validate this approach a simulation environment was developed using a simplified model of the experimental assembly cell consisting of a robot with a gripping device, a queue for part feed, a buffer for part storage and a workplace for the assembly of a target product [20].

A first simulation study investigated the influence of (1) the size of the product to be assembled, (2) the number of parts provided at the queue and (3) the feeding regime (two levels: deterministic supply of needed parts and random supply including unneeded parts) on processing time as well as the number of required pick and place operations (termed MTM-1 cycles). The results of this study show a disproportional increase in processing time with increasing part size and queue length for the deterministic part feed (Fig. 1). Conversely, a stochastic part feed surprisingly leads to a decrease in processing time over the queue length (Fig. 2). However, the number of MTM-1 cycles did not show differences between the

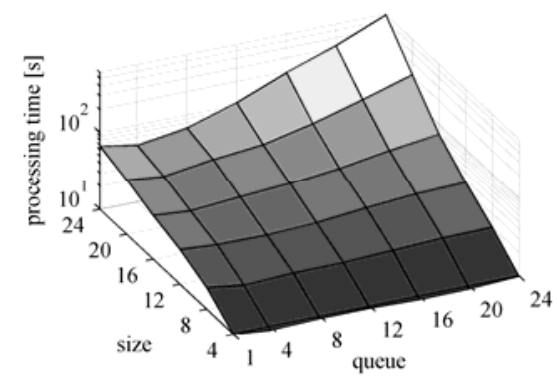

deterministic and stochastic part feed. Mayer et al. [20] further reported that the desired target state was assembled correctly by the CCU in all 8,400 runs. No assembly errors or deadlocks did occur. Therefore, it can be stated that these systems allow for flexible and reliable assembly.

Whereas in the previous conducted studies [20] the feeding regime only differentiated between stochastic and deterministic part feed (in the sense of directly mountable part supply), the focus of the study presented in the following lies on the effect of phaseshifted part feed in case of a queue size of only one part. In case the parts needed in an assembly task are not supplied in the right order, this should have an effect on both dependent variables: processing time as well as performed MTM-1 cycles. The processing time is solely based on simulation time. Hence, no movement time of the elements of the assembly cell is taken into account. In close analogy to the previous introduced studies the following independent variables are defined:

a) size of the target product to be assembled (size): six levels from four to 24 parts in steps of four, whereas no identical parts within the target product occur

b) shape factor (type): between the two extremes of a flat structure of parts (level 1, all parts directly mountable) and a linear structure (level 5, parts only mountable in one particular order), three additional levels are introduced with parts distributed over multiple levels (level 2: 3:1; level 3: 2:2; level 4: $2: 1: 1)$

c) phase-shift (phase): based on the order necessary to build up the linear structure directly, four levels from $0^{\circ}$ (no changes to the order) to $270^{\circ}$ (last $75 \%$ of the original part feed order are moved to start) in steps of $90^{\circ}$ are defined

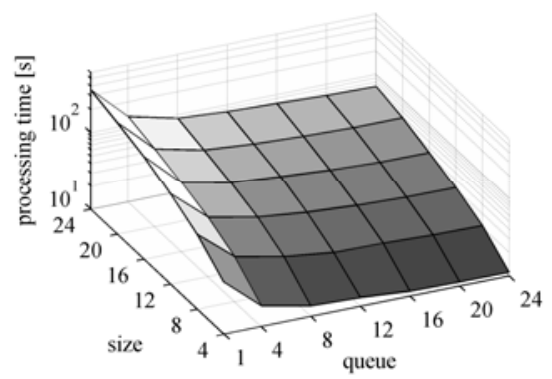

Fig. 1: Processing time of the CCU as a function of number of parts in the target product (size) and number of parts available at the queue (queue) for deterministic part feed (left) and stochastic part feed (right) [20] 
For each combination $(6 \times 5 \times 4)$ of the levels of the independent variables 20 simulation runs were calculated. Self-developed simulation software was used. The runs were scheduled for parallel processing on the high-end Compute Cluster in the Center for Computing and Communication at RWTH Aachen University. For the dependent variables significantly deviate from Gaussian distribution the Sheirer-RayHare test (SRH; [21]) as a nonparametric version of a three-way ANOVA was used with a significance level of $\alpha=0.05$.

The results of the SRH test for the processing time are summarized in Table 1.

Table 1

Results of the three-way SRH test for the dependant variable processing time

\begin{tabular}{llrrr}
\hline source & sum sq. & df & \multicolumn{1}{l}{$\boldsymbol{H}$} & $\boldsymbol{p}$ \\
\hline size & $1,014 \mathrm{E}+09$ & 5 & 2111,266 & $<0,001$ \\
\hline type & $4,047 \mathrm{E}+07$ & 4 & 84,276 & $<0,001$ \\
\hline phase & $5,629 \mathrm{E}+07$ & 3 & 117,215 & $<0,001$ \\
\hline size*type & $7,488 \mathrm{E}+06$ & 20 & 15,594 & 0,741 \\
\hline size*phase & $5,075 \mathrm{E}+06$ & 15 & 10,568 & 0,783 \\
\hline type*phase & $1,709 \mathrm{E}+07$ & 12 & 35,589 & $<0,001$ \\
\hline size*type*phase & $8,824 \mathrm{E}+06$ & 60 & 18,375 & 1,000 \\
\hline error & $2,937 \mathrm{E}+06$ & 2280 & & \\
\hline total & $1,152 \mathrm{E}+09$ & 2399 & & \\
\hline
\end{tabular}

Significant effects can be identified for the independent variable "size" $(H=2111.266 ; d f=5 ; p<0.001)$, "type" $(H=74.317 ; d f=4 ; p<0.001)$ and phase-shift $(H=33.443 ; d f=3 ; p<0.001)$. An interaction effect was found for the factors "type" and "phase" $(H=37.473$; $d f=12 ; p<0.001)$. For the interaction is completely disordinal, the main effects must not be interpreted for the factors "type" and "phase" regarding processing time. Despite the fact, a trend can be seen for the processing time regarding the factors type and phase-shift (Figure 3). For a phase-shift of $0^{\circ}$, no influence due to the shape factor (type) can be seen. The processing time is approximately proportional to the size of the target part. With increasing phase-shift, the influence of the shape becomes more evident. Whereas the increase in processing time compared to type 1 does not show great differences between type 2, type 3 and type 4 for the phase-shifts $(\sim 12 \%$ on average), an average increase in processing of $30 \%$ at $90^{\circ}$ phase-shift, $55 \%$ at $180^{\circ}$ phase-shift and $78 \%$ at $180^{\circ}$ phase-shift was observed for type 5 .
The results of the SRH test for the MTM-1 cycles are displayed in Table 2.

Table 2

Results of the three-way SRH test for the dependant variable MTM-1 cycle

\begin{tabular}{llrrr}
\hline source & sum sq. & \multicolumn{1}{l}{ df } & \multicolumn{1}{l}{$\boldsymbol{H}$} & \\
\hline size & $1,056 \mathrm{E}+09$ & 5 & 2213,723 & $<0,001$ \\
\hline type & $3,546 \mathrm{E}+07$ & 4 & 74,317 & $<0,001$ \\
\hline phase & $1,595 \mathrm{E}+07$ & 3 & 33,443 & $<0,001$ \\
\hline size*type & $8,508 \mathrm{E}+06$ & 20 & 17,835 & 0,598 \\
\hline size*phase & $3,475 \mathrm{E}+06$ & 15 & 7,284 & 0,949 \\
\hline type*phase & $1,788 \mathrm{E}+07$ & 12 & 37,473 & $<0,001$ \\
\hline size*type*phase & $7,121 \mathrm{E}+06$ & 60 & 14,926 & 1,000 \\
\hline error & $0,000 \mathrm{E}+00$ & 2280 & & \\
\hline total & $1,145 \mathrm{E}+09$ & 2399 & & \\
\hline
\end{tabular}

Significant effects can be identified for the independent variable "size" $(H=2213.723 ; d f=5 ; p<0.001)$, "type" $(H=84.276 ; d f=4 ; p<0.001)$ and phase-shift $(H=117.215 ; d f=3 ; p<0.001)$. An interaction effect was found for the factors "type" and "phase" $(H=35.589 ; d f=12 ; p<0.001)$. For "phase" shows disordinal behavior regarding "type", but "type" is ordinal to "phase", the interaction is hybrid. Hence, the main effect of "phase" can be interpreted. Similar to the results for the processing time, no influence on MTM-1 cycles due to the shape factor (type) can be seen for a phase-shift of $0^{\circ}$. The number of MTM-1 cycles is proportional to the size of the target part. Regarding the increase of MTM-1 cycles compared to type 1 no differences between type 2, type 3 and type 4 can be observed for the phase-shifts $(\sim 12 \%$ on average). Conversely, an average increase in the number of MTM- 1 cycles of $25 \%$ at $90^{\circ}$ phase-shift, $50 \%$ at $180^{\circ}$ phase-shift and $75 \%$ at $180^{\circ}$ phase-shift was observed for type 5 .

The presented simulation studies partially support the findings of Mayer et al. [20]. It could be shown, that the CCU is able to react to changes in part feed, for the observed number of MTM-1 cycles did not exceed theoretical expectations and further corresponds to the observed simulation time of the CCU. In contrast to the aforementioned studies the part feed can be considered deterministic, as only need parts were contained. However, the difference here lies in the target state (which consisted of maximal number of different parts) as well as the queue size (only one part at a time). In case of increased number of parts on the queue a disproportional increase in processing time is to be expected. 

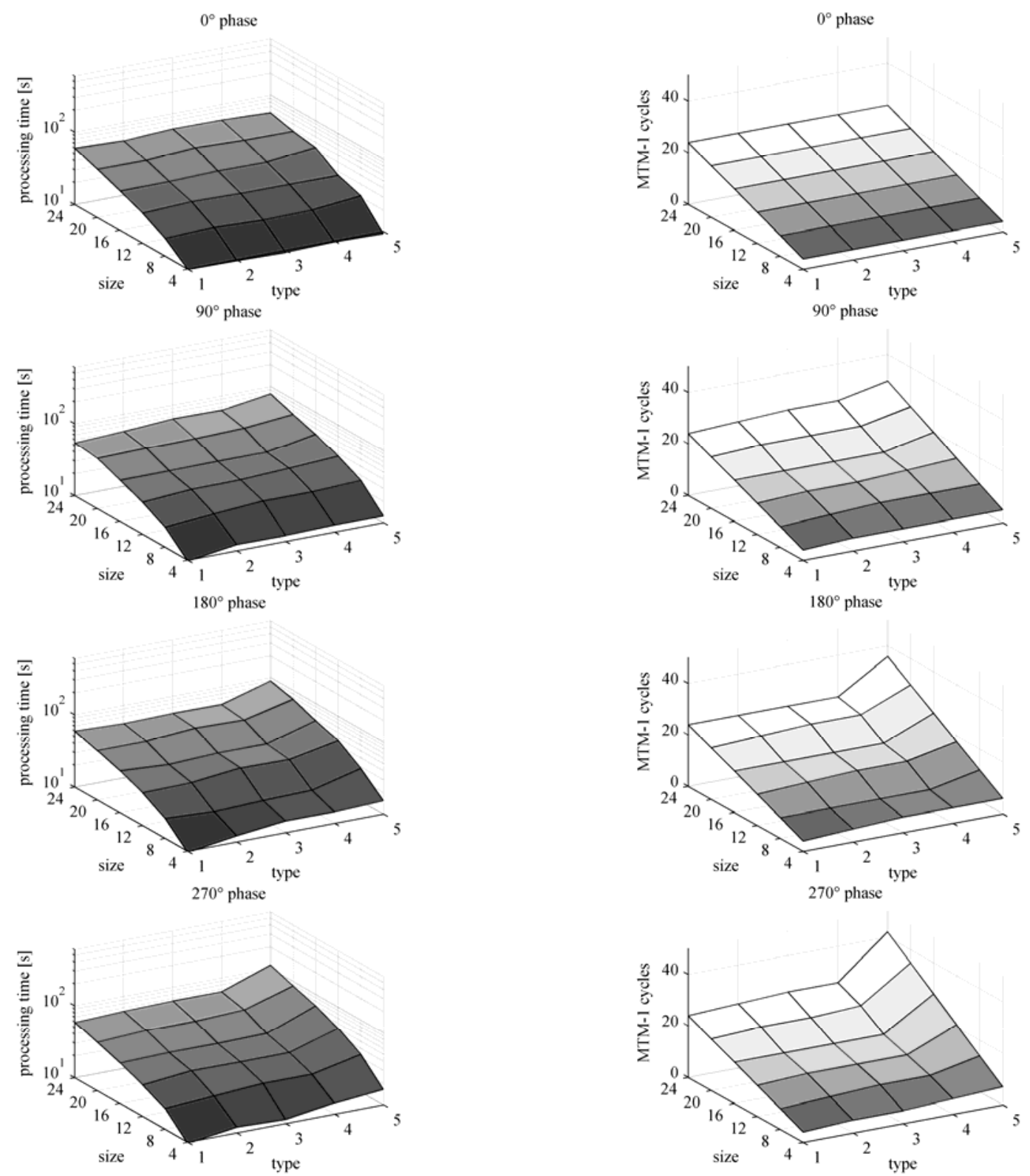

Fig. 3: Dependent variables processing time (left) and MTM-1 cycles (right) as a function of the independent variables "phase", "size" and "type"

\section{Summary and Outlook}

Especially in highly automated manufacturing systems that are aiming at producing products in almost any variety in product space, an increase in conventional automation will not necessarily lead to a sig- nificant increase in productivity. Therefore, novel concepts towards proactive, agile and versatile manufacturing systems have to be developed. Cognitive automation is a promising approach to improve proactive system behavior as it could be shown by the results presented within this paper.

To be able to accomplish complex assembly tasks without impairing the $\mathrm{CCU}$ with calculations that 
cannot be solved in polynomial time, future investigations will focus on a hybrid approach [22] where the predefined planning problem is solved prior to the assembly by generating a state graph [23] that describes all possible assembly sequences for the intended product. This graph can also be updated during assembly. The reasoning component within SOAR uses this state graph to adapt the plan to the actual state of the assembly and part supply.

\section{Acknowledgment}

The authors would like to thank the German Research Foundation (DFG) for its kind support of the research on cognitive automation within the Cluster of Excellence Integrative Production Technology for High-Wage Countries.

\section{References}

[1] Kinkel S, Friedwald M, Hüsing B, Lay G, Lindner R (2008) Arbeiten in der Zukunft, Strukturen und Trends der Industriearbeit. Studien des Büros für Technikfolgen-Abschätzung bei Deutschen Bundestag - 27. edition sigma, Berlin (in German)

[2] Bainbridge L (1987) Ironies of Automation. In: Rasmussen J, Duncan K, Leplat J (Eds) New Technology and Human Error, Wiley, Chichester

[3] Onken R, Schulte A (2010) System-ergonomic design of cognitive automation. Studies in Computational Intelligence 235. Springer, Berlin

[4] Kempf T, Herfs W, Brecher C (2008) Cognitive Control Technology for a Self-Optimizing Robot Based Assembly Cell. In: Proceedings of the ASME 2008 International Design Engineering Technical Conferences \& Computers and Information in Engineering Conference, America Society of Mechanical Engineers, U.S.

[5] Karim S, Sonenberg L, Tan AH (2006) A Hybrid Architecture Combining Reactive, Plan Execution and Reactive Learning. In: Proceedings of the 9th Biennial Pacific Rim International Conference on Artificial Intelligence (PRICAI), China

[6] Gat E (1998) On Three-Layer Architectures. In: Kortenkamp D, Bonnasso R, Murphy R (Eds) Artificial Intelligence and Mobile Robots. AAAI Press, pp 195-211

[7] Russell SJ, Norvig P (2003) Artificial Intelligence: A Modern Approach. Pearson Education

[8] Paetzold K. (2006) On the importance of a functional description for the development of cognitive technical systems. In: International Design Conference 2006, Dubrovnik

[9] Mayer M, Odenthal B, Ewert D, Kempf T, Behnen D, Büscher C, Kuz S, Müller S, Hauck E, Kausch B, Schilberg D, Herfs W, Schlick C, Jeschke S, Brecher C (2011) Self-optimising assembly systems based on cognitive technologies. In:
Brecher C (Ed) Integrative Production Technology for HighWage Countries (in press)

[10] Mayer M, Odenthal B, Faber M, Neuhöfer J, Kabuß W, Kausch B, Schlick C (2009) Cognitive Engineering for Direct Human-Robot Cooperation in Self-optimizing Assembly Cells In: Kurosu M (Ed) Human Centered Design, Lecture Notes in Computer Science 5619. Springer, Heidelberg, pp 1003-1012

[11]Lehman J, Laird J, Rosenbloom P (2006) A gentle introduction to soar, an architecture for human cognition: 2006 update. Retrieved 17 May 2010 from http://ai.eecs.umich.edu/soar/ sitemaker/docs/misc/GentleIntroduction-2006.pdf

[12] Mayer M, Odenthal B, Grandt M, Schlick C (2008) Anforderungen an die benutzerzentrierte Gestaltung einer Kognitiven Steuerung für Selbstoptimierende Produktionssysteme. In: Gesellschaft für Arbeitswissenschaft eV (Ed) Produkt- und Produktions-Ergonomie - Aufgabe für Entwickler und Planer. GfA-Press, Dortmund (in German)

[13] Gazzola V, Rizzolatti G, Wicker B, Keysers C (2007) The anthropomorphic brain: The mirror neuron system responds to human and robotic actions. NeuroImage 35:1674-1684

[14] Mayer M, Odenthal B, Grandt M, Schlick C (2008) TaskOriented Process Planning for Cognitive Production Systems using MTM. In: Karwowski W, Salvendy G (Eds) Proceedings of the 2nd International Conference on Applied Human Factors and Ergonomic (AHFE) 14.-17. July 2008. USA Publishing, Las Vegas, pp 1-9

[15]Hauck E, Gramatke A, Henning K (2008) Cognitive Technical Systems in a Production Environment. In: Proceedings of the Fifth International Conference on Informatics in Control, $\mathrm{Au}$ tomation and Robotics. ICINCO, Madeira, Portugal

[16] Wiendahl HP, ElMaraghy HA, Nyhuis P, Zäh MF, Wiendahl HH, Duffie N, Brieke M (2007) Changeable Manufacturing. Classification, Design and Operation. In: Annals of the CIRP 56(2):783-809

[17] Hoffmann J (2001) FF: The Fast-Forward Planning System. In: The AI Magazine

[18]Hoffmann J, Brafman R. (2005) Contingent Planning via Heuristic Forward Search with Implicit Belief States. In: Proceedings of the 15th International Conference on Automated Planning and Scheduling, Monterey, CA, USA

[19]Castellini C, Giunchiglia E, Tacchella A (2001) Improvements to sat-based conformant planning. In: Proc. of the 6th European Conf. on Planning

[20] Mayer M, Schlick C, Ewert D, Behnen D, Kuz S, Odenthal B, Kausch B (2011) Automation of robotic assembly processes on the basis of an architecture of human cognition. In: Production Engineering Research and Development (DOI 10.1007/s11740-011-0316-z)

[21] Scheirer J, Ray WS, Hare N (1976) The Analysis of Ranked Data Derived from Completely Randomized Factorial Designs. Biometrics 32(2):429-434

[22] Ewert D, Mayer M, Kuz S, Schilberg D, Jeschke S (2010) A hybrid approach to cognitive production systems. In: Proceedings of the 2010 International Conference on Intelligent Robotics and Applications (ICIRA 2010) Shanghai, China

[23]Zaeh MF, Wiesbeck M (2008) A Model for Adaptively Generating Assembly Instructions Using State-based Graph. In: Mitsuishi M, Ueda K, Kimura F (eds) Manufacturing Systems and Technologies for the New Frontier. Springer, Berlin 